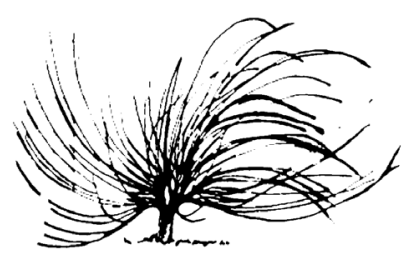

\title{
Aplicabilidad de las pedagogías emergentes en el e-learning
}

\author{
Francisco Mora-Vicarioli ${ }^{1}$ \\ Universidad Estatal a Distancia \\ Costa Rica \\ fmora@uned.ac.cr \\ Kathya Salazar-Blanco ${ }^{2}$ \\ Universidad Estatal a Distancia \\ Costa Rica \\ ksalazarb@uned.ac.cr
}

\section{Resumen}

Este artículo es el resultado del interés por realizar un aporte actual al tema de las pedagogías emergentes y su aplicabilidad al contexto del e-learning. El término "emergente" no tiene que ver con una nueva pedagogía, sino con aquella que se adapta a las potencialidades que ofrecen las tecnologías de la información y comunicación (TIC) para los procesos académicos. Anivel metodológico, para esta investigación se realizó un análisis documental en más de 60 fuentes académicas, que tuvo, en primera instancia, el propósito de obtener las categorías que están involucradas en las pedagogías emergentes, con el fin de facilitar el proceso hermenéutico; posterior a esto, se hizo una búsqueda exhaustiva de exponentes sobre los diferentes temas involucrados en las pedagogías

Recibido: 27 de abril de 2018. Aprobado: 10 de abril de 2019.

http://dx.doi.org/10.15359/rep.14-1.6

1 Máster en Tecnología Educativa de la UNED de Costa Rica. En la actualidad, productor académico Programa de Aprendizaje en Línea en la Universidad Estatal a Distancia.

2 Licenciada en Informática Educativa de la Universidad Estatal a Distancia y Máster en Tecnología Educativa del Tecnológico de Monterrey- México. En la actualidad, productora académica del Programa de Aprendizaje en Línea de la Universidad Estatal a Distancia. 
emergentes: aprendizaje ubicuo, personalismo educativo, aprendizaje colaborativo, aprendizaje contextual o situado y evaluar para aprender. Se buscó dar un aporte al conocimiento del tema y, en particular, al rol que debe asumir el personal docente y posibles retos que enfrenta por medio de las pedagogías emergentes, donde se destacan las competencias en el manejo de las TIC y de la mediación pedagógica como elementos primordiales, aunado a promover un rol activo en el estudiantado.

Palabras clave: Educación a distancia, tecnologías de la información y comunicación (TIC), e-learning, u-learning, pedagogías emergentes, personalismo educativo, e-actividades, aprendizaje basado en problemas, aprendizaje basado en proyectos, trabajo colaborativo, evaluar para aprender, ecologías del aprendizaje.

\begin{abstract}
This article is the result of the interest in making a current contribution to the topic of emerging pedagogies and their applicability to the context of e-learning. The term "emergent" does not have to do with a new pedagogy, but with one that adapts to the potential offered by information and communication technologies (ICT's) for academic processes. At the methodological level, for this research, a documentary analysis was carried out in more than 60 academic sources, which had, at first, the purpose of obtaining the categories that are involved in emerging pedagogies, in order to facilitate the hermeneutical process, after this an exhaustive search was made of exponents about the different topics involved in emerging pedagogies: ubiquitous learning, educational personalism, collaborative learning, contextual or situated learning and evaluating to learn. We sought to contribute to the knowledge of the subject and in particular to the role that the teacher should assume and possible challenges that are faced through emerging pedagogies, in which skills in the management of ICT and pedagogical mediation are highlighted as key elements, in addition to promoting an active role in the students.
\end{abstract}


Keywords: distance education, information and communication technologies (ICT's) e-learning, u-learning, emerging pedagogies, educational personalism, e-activities, problem-based learning, project-based learning, collaborative work, assessment for learning, ecologies of learning

\section{Introducción}

Con la incorporación de las tecnologías de la información y la comunicación (TIC) en el ámbito educativo surge el e-learning, el cual consiste en la creación e implementación de estrategias pedagógicas innovadoras apoyadas con las TIC que aportan beneficios al proceso educativo. San Martín (2009) reflexiona sobre cómo estas tecnologías permean los pilares de la enseñanza, además señala que la sola presencia de ellas genera gran interés en profesionales de la educación y en estudiantes, esto hace que se deban revisar las prácticas actuales de enseñanza, además de la evolución que deben llevar hacia el uso de todo el potencial de las TIC.

El e-learning, como resultado de una tendencia formativa actual en instituciones de educación a distancia, presenta una serie de áreas de oportunidad en cuanto a flexibilidad, acompañamiento e interacción entre estudiantes y docentes, sin dejar de lado la evaluación de los aprendizajes, todo lo anterior, desde un rol más activo por parte del estudiantado.

Las nuevas tendencias, dentro del desarrollo de la tecnología educativa, apuntan hacia un incremento de las actividades basadas en la interacción y la creación colectiva de conocimientos. Estrategias como la resolución de problemas, pensamiento crítico, razonamiento y reflexión pueden ser exitosamente implementadas para la construcción del conocimiento, mediante la creación de entornos de interacción social a través de herramientas para el trabajo colaborativo en red. (Avello y Duart, 2016, p. 273)

Como lo aportan Ilgaz y Gulbahar (2017), la preferencia por el e-learning tiene que ver con elementos como la tecnología utilizada y el tipo de pedagogía que se aplica, además de la accesibilidad y flexibilidad que aporta lo virtual en los procesos educativos. 
Actualmente los escenarios de formación virtual se encuentran sometidos a una evolución producida no sólo por la incorporación de herramientas y recursos tecnológicos, sino también en cuanto a lo pedagógico como producto de nuevos roles de los actores que se están configurando en dichos espacios. (Morales y Munévar, 2014, p. 80)

La virtualidad representada por el e-learning resulta un campo de gran interés, por cuanto sus opciones de colaboración, comunicación y evaluación permiten grandes innovaciones y una gran cercanía entre estudiantes y profesorado.

De acuerdo con lo anterior, las prácticas pedagógicas se ven permeadas por los cambios económicos, políticos y culturales, por lo cual resulta importante reflexionar sobre el sentido del aprendizaje en la sociedad del conocimiento (Ricaurte, 2016), por tanto, los objetivos planteados para esta investigación consisten en analizar el rol de las pedagogías emergentes en el e-learning y las diferentes tendencias que promueven las pedagogías emergentes. Estos objetivos se enmarcan en debatir y reflexionar acerca del impacto de las pedagogías emergentes en el campo del e-learning, así como abrir nuevas preguntas sobre la temática. La investigación, a su vez, trata de brindar un soporte teórico amplio y actual sobre el tema y las categorías que surgen de él.

\section{Marco teórico}

En la actualidad la dupla educación y TIC es casi indivisible, en el sentido de que tanto en la educación presencial como en la educación a distancia la diversidad de dispositivos, la amplia gama de herramientas para uso online y offline, la posibilidad de almacenamiento en la nube y la variedad de aplicaciones hacen que docentes y estudiantes busquen constantemente formas de implementación que faciliten y flexibilicen procesos de comunicación, investigación y de colaboración en trabajos asignados.

Ante este panorama, Roblizo y Cózar (2015) señalan que hoy concebir la pedagogía y la educación sin la presencia de las tecnologías de la información y comunicación es algo impensable.

Desde la perspectiva docente se identifica claramente que: "reconocen las TIC como herramientas que ofrecen la posibilidad de cambiar los procesos; y que, las TIC permiten flexibilizar los procesos 
educativos y facilitan así la atención a la diversidad de los alumnos" (Fernández, 2016, p. 41).

Ante este tipo de reconocimiento, el uso de las TIC desde una visión pedagógica hace que el personal docente busque nuevas tendencias en la implementación de estas, sin dejar de lado que el rol estudiantil también varía en función de las tecnologías. Además, se puede ejercer una realimentación formativa más expedita, por ello resulta de gran interés investigar acerca del tipo de prácticas educativas y cambios que surgen en este contexto.

Una de estas prácticas son las pedagogías emergentes, que involucran términos ya introducidos en el ámbito internacional como el personalismo educativo, aprendizaje ubicuo (u-learning), trabajo colaborativo, aprendizaje situado o contextual, aprendizaje basado en problemas, evaluar para aprender, entre otros.

Gros (2015) señala:

Las pedagogías emergentes surgen en los contextos de la sociedad del conocimiento en red, se basan en la integración de las tecnologías digitales, la exploración y la modificación de las pedagogías existentes y desarrollo de nuevas propuestas teóricas y prácticas (p. 63).

Las pedagogías emergentes tratan de reinterpretar lo que la tecnología puede lograr en los procesos educativos y el tipo de mediación o adaptación de las prácticas educativas que se requiere por medio del uso de las TIC y, particularmente, cómo podemos adaptar mejor los procesos para que se ajusten a las necesidades del estudiantado.

Las pedagogías emergentes surgen con un aporte claro: variar el rol docente y estudiantil, el hecho de contextualizar y poder visualizar sus beneficios por medio del uso de las TIC y, en particular, por medio del e-learning.

La sociedad del conocimiento alimenta amplias posibilidades educativas, cambios de prácticas y aprovechamiento de recursos tecnológicos; todo ello es el principal motor que hace más evidentes las pedagogías emergentes. 


\section{La pedagogía}

En toda su complejidad y vista como un proyecto o proceso, la pedagogía se refiere a los contenidos, métodos, estrategias, así como a los esquemas metodológicos y estratégicos; implica el ¿qué?, ¿cómo? y ¿con qué?, esto es, tener claridad de lo que se hará, la forma en la que se hará y los recursos a utilizar en el quehacer educativo.

Si queremos considerar la pedagogía como una disciplina científica, al igual que en el resto de las ciencias, necesitamos una constante reflexión sobre la lógica de la investigación a partir de los desarrollos conceptuales en este campo y de la práctica de investigación educativa. (De Miguel, 2015, p. 276)

La pedagogía requiere de investigación en la práctica educativa, lo que implica que el personal docente realice procesos metodológicos para reflexionar sobre su papel y el propósito de este, para, a su vez, ajustarlos a las necesidades de la población meta.

Según lo planteado, la pedagogía resulta una disciplina amplia, en cuanto a las relaciones que establece por medio de las prácticas pedagógicas y sus estrategias. Como vemos, esta disciplina brinda una guía en el quehacer docente (Jiménez, 2010). Sobre la presencia de la pedagogía en diferentes contextos, la visión de Giroux (2003) resulta orientadora:

Como práctica activa, la pedagogía está en todos estos espacios públicos en los que la cultura opera para asegurar las identidades, hace de puente para negociar la relación entre el conocimiento, placer y valores, y rinde autoridad crucial y problemática al legitimar prácticas sociales determinadas, comunidades y formas de poder. (p. 4)

De acuerdo con lo anterior, las estrategias de la pedagogía son muy amplias:

Precisa elaborar planteamientos que orienten en el diseño de estos nuevos espacios a través de experiencias basadas en la interacción social, la participación activa y los entornos complejos. Cuatro aspectos básicos centran las estrategias de formación: la 
personalización, el aprendizaje activo, el aprendizaje colaborativo y el aprendizaje autónomo o autodirigido. (Gros, 2015, pp. 62-63)

\section{El estudiante y el e-learning}

En la elaboración de estos planteamientos y en el surgimiento de nuevos espacios de educación situada, en el contexto del uso de las TIC, y más propiamente de la virtualidad, emerge un término de uso generalizado a nivel mundial: el e-learning.

El e-learning, o aprendizaje electrónico o virtual, se considera un fenómeno global, por una variedad de fuerzas sociales, económicas y tecnológicas, así como por las demandas de estudiantes (Shah, Attid y ur Rheman, 2017).

Con el avance de las tecnologías de la información y la comunicación, así como con la implementación del e-learning, surgen las posibilidades de migrar a un paradigma de diseño instruccional pasando de un diseño centrado en el alumnado a uno que consiste en muchos diseños para cada estudiante, los cuales deben ser clasificados según los estilos de aprendizaje y las preferencias estudiantiles tendientes a entornos de aprendizaje electrónico personalizado (Alhawiti y Abdelhamid, 2017).

El e-Learning ahora está siendo reconocido, y preferido, por más y más personas, lo que resulta en un mayor número de estudiantes a distancia en los últimos años. Numerosos estudios de investigación se centran en las preferencias del alumno para el aprendizaje en línea, la mayoría convergiendo en torno a las características y diferencias individuales, así como a las características de la tecnología y pedagogía utilizada. (IIgaz y Gulbahar, 2017, p. 130)

Dentro de estos escenarios educativos con apoyo de nuevas tecnologías, el papel del estudiantado se hace más protagónico dando lugar a "estudiantado virtual", o aquel que desarrolla sus procesos académicos en el e-learning.

Es importante conocer que esta figura posee características particulares y necesidades bien definidas, en cuanto a espacios de formación que permitan combinar otras ocupaciones (vida laboral, familiar, entre otras), contenidos en formatos atractivos, actividades flexibles y 
posibilidades comunicativas, tanto sincrónicas (en tiempo real) como asincrónicas, siendo así el e-learning debe satisfacer las demandas estudiantiles de hoy y garantizar beneficios acordes con sus necesidades.

Tal como lo aporta Silva (2017), refiriéndose a la nueva generación de estudiantes, "vive la tecnología como parte de su entorno habitual, para ellos la información y el aprendizaje no están relegados a los muros del aula, ni es ofrecida en forma exclusiva por el profesor" (p. 2). En relación con el perfil de los nuevos grupos de estudiantes, debe existir un abordaje distinto por parte del personal docente, el cual fungirá más bien como facilitador y guía.

En cuanto a los beneficios del $e$ - learning para el estudiantado, Dorego (2016) menciona los siguientes: "el nivel de inmediatez, así como de interacciones; las posibilidades de acceso a los cursos desde cualquier lugar y tiempo; y la capacidad de retorno de comentarios y de discusión que ayudan a la construcción del aprendizaje por el propio alumno" (p. 5).

En una investigación realizada por Camacho, Gómez y Pintor (2015), se determinaron algunas competencias necesarias que debe poseer el estudiantado para un ambiente educativo virtual. Ellas son:

- Manejo de información para llevar a cabo la investigación, análisis y síntesis de lo relevante de los temas a estudiar y evitar el plagio.

- $\quad$ Administración del tiempo: uso eficiente de la agenda de sus tiempos, para que destine los adecuados a sus diversas actividades.

- Comunicación mediante las TIC: manejar las herramientas de mensajería interna, foros, correos electrónicos, así como la adecuada redacción y expresión. (p. 22)

La consideración dada con respecto a las competencias permite tener en cuenta la adaptación para la población, según sus particularidades y perfil de ingreso.

Desde una perspectiva general, Velazco, Abuchar, Castilla y Rivera (2017), señalan algunos beneficios que son parte del éxito del e-learning: reducción de costos, rapidez y agilidad en cuanto a la comunicación; acceso, flexibilidad de agenda, interacción y colaboración.

Cabe también señalar que una de las bondades del e-learning es que puede incluir el elemento de la presencialidad para el desarrollo de actividades que requieren la presencia de estudiante o de docente, A esta 
modalidad educativa se le conoce con el nombre de blended-learning $o$ aprendizaje semipresencial, debido a la combinación de elementos de la educación virtual con la educación presencial.

El b-learning, al combinar prácticas comunes de educación presencial con el aprendizaje en línea, resulta una alternativa valiosa para la población con algún tipo de discapacidad, pues brinda mayor accesibilidad a los procesos de formación, ya que a través de la asistencia, a cada estudiante se le puede valorar habilidades y destrezas difíciles de conocer en ambientes virtuales; el b-learning da una nueva visión al enfoque de pedagogía tradicional donde el rol estudiantil es más activo (Cervantes y Saker, 2016).

\section{Pedagogía en entornos virtuales (tutor virtual)}

En el apartado anterior vemos que con el reconocimiento del e-learning como una modalidad educativa de grandes posibilidades, el rol estudiantil juega un papel más protagónico, de la misma forma el papel docente debe serlo, sin que exista preeminencia entre uno y otro roles. Pero es importante tener claro que la práctica pedagógica en entornos de aprendizaje virtual o e-learning debe desarrollarse de manera distinta, y que el personal tutor, además de las competencias pedagógicas que per se debe poseer cada docente, debe adquirir también competencias tecnológicas; ello, por cuanto estas deben y tienen que ser diferentes a las que se emplean en entornos de aprendizaje presencial o a distancia.

En cuanto a las competencias didácticas - metodológicas, Suárez, Almerich, Díaz y Fernández (2012) indican que estas se refieren al conjunto de conocimientos y habilidades que facultan al personal docente para emplear esos recursos tecnológicos en el diseño y desarrollo del currículo, para saber qué, cuándo y cómo ha de utilizar los recursos para la planificación y organización de su práctica educativa.

En relación con las competencias tecnológicas, estos mismos autores las definen como el conjunto de conocimientos y habilidades que el personal docente debe poseer para incorporar el uso correcto de las herramientas tecnológicas en su quehacer educativo. La adquisición y desarrollo de estas competencias le permitirá alcanzar la "alfabetización tecnológica", la cual consiste en la competitividad para hacer uso de las tecnologías de la información y la comunicación, según su funcionalidad, para seleccionarlas y aplicarlas en el 
diseño de ambientes de aprendizaje acordes con las necesidades educativas (Area y Guarro, 2012).

A la luz de lo anterior, sin duda alguna, la instrucción y la mediación en ambientes virtuales de aprendizaje e-learning requieren el ejercicio de una pedagogía distinta a la tradicional. Ante esta premisa se hace necesario resaltar cuáles son las funciones del personal tutor en ambientes e-learning.

Badia, Meneses y García (2018) aportan los roles, enfoques y atributos de la enseñanza virtual o e-learning, en relación con el personal docente, el estudiantado y la forma en que se efectúa el proceso académico; en general, la innovación y mediación que se realice en el e-learning son las claves para el éxito.

Un docente en un entorno virtual exige preparación, actuaciones y actitudes distintas, que, en definitiva, se concretan en saber acompañar a través de la red a cada estudiante a lo largo de su aprendizaje. Esta es la función clave del docente en estos escenarios, sin dejar de lado las otras funciones implícitas en toda tarea docente. (Morales y Munévar, 2014, p. 86)

La reflexión brindada implica que el personal docente cuente con competencias tecnológicas, las cuales se logran por medio de capacitación y experiencia en el uso de los entornos virtuales de aprendizaje, tanto desde el rol docente como del estudiantil, experiencias que pueden brindar un referente valioso y permitirán tomar decisiones importantes en cuanto a mediación en cualquier ambiente de aprendizaje.

Además de lo anterior, entre las funciones del personal tutor virtual se pueden considerar las siguientes:

1. Con habilidades comunicativas.

2. Motivador.

3. Dinámico y con habilidades para crear, dentro del contexto, espacios de comunicación que permitan la interacción.

4. Facilitador del aprendizaje mediante estrategias didácticas utilizando distintos recursos y materiales exclusivos para ambientes virtuales. 
Ahora bien, el personal docente como mediador de situaciones de aprendizaje debe tener la capacidad para hacer una integración total de sus competencias didácticas, metodológicas y tecnológicas para crear estos espacios de aprendizaje acordes con las demandas y necesidades educativas que corresponden a su ejercicio profesional; esto va de la mano con lo que señalan las pedagogías emergentes, que más adelante se describirán.

\section{Tipos de aprendizaje en $e$-learning}

El cambio de los escenarios de formación, desde lo presencial, pasando por la educación a distancia y el e-learning, brindan una pista de la evolución de los procesos educativos y cómo el cuerpo docente debe considerar todo esto para su desempeño profesional. A continuación, se describen algunos de estos escenarios.

\section{a. Aprendizaje ubicuo (u-learning)}

El aprendizaje ubicuo es señalado por algunas autorías, como Quintero y Romero (2018), como un nuevo modelo pedagógico, caracterizado por su permanencia, accesibilidad, inmediatez, actividades situadas (contextualizadas), adaptabilidad.

Por otro lado, Jenaro, Flores, González y Martínez (2013) orientan sobre la importancia de que el personal docente proponga metodologías más activas, como el aprendizaje colaborativo y el uso de actividades contextuales o aprendizaje situado.

El aprendizaje ubicuo se relaciona con el término de aprendizaje expandido, el cual se aproxima a un aprendizaje abierto, colaborativo, democrático y comprometido, a su vez; propicia habilidades blandas o competencias de aprendizaje para toda la vida y la futura ciudadanía (Díez y Díaz, 2018). Además, el aprendizaje ubicuo aporta en el contexto educativo:

Prepara y alienta a los estudiantes a continuar aprendiendo toda su vida, enseñándoles cómo utilizar los recursos disponibles para acceder a la información cuando esta es necesaria y desarrollando sus habilidades para buscarla e interpretarla. (Quintero y Romero, 2018, p. 16) 
Para Velandia, Serrano y Martínez (2017), "los ambientes de aprendizaje ubicuos facilitan el aprendizaje contextual si se les proporciona el contenido apropiado, en el momento adecuado y en el lugar indicado" (p. 16), más adelante se describe el aprendizaje contextual como una tendencia que permite fomentar el aprendizaje activo y significativo.

Quintero y Romero (2018), refiriéndose al aprendizaje ubicuo, en relación a la forma en que este ocurre, señalan:

El concepto se relaciona directamente con aprender cualquier cosa, en cualquier momento y en cualquier lugar utilizando tecnologías e infraestructuras de informática ubicua. Uno de los objetivos últimos de la enseñanza es incrementar la calidad de nuestra vida diaria. (p. 15)

Se puede inferir que el aprendizaje ubicuo utiliza las TIC para poder brindar espacios de formación, en este caso, la portabilidad de los diferentes dispositivos (teléfonos inteligentes y tabletas) aporta para que se lleve a cabo este tipo de aprendizaje. Por otra parte, para Flores y García (2017):

La tecnología móvil ofrece la posibilidad de estar conectado en cualquier momento y desde cualquier lugar. Cada vez hay más dispositivos que funcionan como ordenadores: teléfonos móviles inteligentes, televisores inteligentes, videocámara, cámara de fotos, tabletas, entre otros. Estas plataformas se encuentran interconectadas y han sido nombradas con el término computación ubicua. (p. 28)

\section{b. Aprendizaje colaborativo}

En el contexto del e-learning, los trabajos colaborativos, permiten que cada estudiante realice aportes en función de roles asignados, conocimientos y competencias particulares; además, es posible trabajar ejes transversales de diálogo, negociación y tolerancia. Así lo respalda una investigación efectuada por Mora y Hooper (2016) donde determinan una inclinación por parte del estudiantado hacia las actividades colaborativas y cómo estas pueden favorecer los estilos de aprendizaje. 
Una característica de este tipo de aprendizaje es el nivel de actividad y de responsabilidad que debe aportar el estudiantado durante el proceso educativo. A partir de lo anterior, Hernández, González y Muñoz (2015) indican que con el aprendizaje colaborativo "se estimula el desarrollo de competencias transversales necesarias para el aprendizaje en comunidad y para aprender a lo largo de toda la vida" (p. 151).

En cuanto a la labor docente, Avello y Duart (2016) señalan que este rol en el aprendizaje colaborativo tiene que ver con el monitoreo, por cuanto permite el seguimiento y guía, particularmente con el fin de propiciar el éxito en este tipo de dinámicas en donde la función docente es mediar y facilitar la experiencia de aprendizaje que, en conjunto, debe realizar el estudiantado.

Para Avello y Duart (2016), algunas recomendaciones para la efectiva aplicación del aprendizaje colaborativo son: formación de los grupos, selección de la tarea grupal, claridad y flexibilidad de la tarea, significatividad de la tarea, monitoreo y control de la tarea.

\section{c. Aprendizaje contextual o situado}

Para De Corte (2015): "La perspectiva del aprendizaje situado enfatiza que este se realiza esencialmente con la interacción y, especialmente, a través de la participación en actividades y contextos sociales y culturales" (p. 11), la interacción es precisamente donde el aprendizaje colaborativo y el aprendizaje contextual convergen para generar los beneficios ya señalados.

Ahora bien, para De Corte (2015), el aprendizaje contextual o situado es tendiente a lograr mayor significatividad en la formación del estudiantado, pues implica visualizar elementos de aplicación para la vida cotidiana, a la vez que fomenta competencias para la vida, lo cual es denominado como competencia de adaptación. Algunos elementos cognitivos necesarios para adquirir esta competencia son: conocimiento específico, estrategias de análisis, destrezas autorreguladoras, actitudes positivas sobre campos académicos. Desde la perspectiva de Castillo y Cerón (2015): "La enseñanza situada tiene como finalidad propiciar el aprendizaje autónomo y crítico, y aunque el estudiante recibe una acción tutorial del docente para aprender a hacer, es él quien tiene que aprender a reflexionar sobre su propio hacer" (p. 36). 
En general, el aprendizaje situado es tendiente a obtener mayor provecho de las actividades académicas confiriéndoles mayor significado, para que el estudiantado logre un aprendizaje crítico. Cabe señalar que el aprendizaje situado tiene gran aplicación en la enseñanza de las ingenierías y las ciencias médicas, pero es reconocida su relevancia para otras áreas disciplinares.

\section{d. Aprendizaje basado en problemas (ABP)}

El aprendizaje basado en problemas, o más conocido por sus siglas de ABP, según Martín, Díaz y Sánchez (2015) es:

Una metodología que permite formar grupos de trabajo a los que se les presenta un problema a resolver buscando información, analizando ésta y proponiendo soluciones. Los alumnos se enfrentarán a un problema real del área de operaciones de una empresa. Deberán investigar, valorar alternativas, aplicar técnicas estadísticas y tomar decisiones que resuelvan los problemas planteados. (p. 165)

Además, Martín, Díaz y Sánchez (2015) mencionan que está relacionado con el aprendizaje interdisciplinar y con el aprendizaje situado o contextual, el cual siempre será más provechoso que actividades centradas en una sola área de estudio.

Roca, Reguant y Canet (2015) aportan también que el ABP resulta un método centrado en el estudiantado, donde este es el actor principal que debe asumir un rol activo; el personal docente, por su parte, resulta ser guía o facilitador.

Rodríguez y Fernández (2017) lo definen:

Es una didáctica específica en la que el proceso de enseñanza y aprendizaje está caracterizado por el enfrentamiento de los alumnos a problemas más o menos complejos, reales las más de las veces, y para lo cual podrán disponer de cuanto material consideren necesario. (p. 62) 
En la misma línea, Rodríguez y Fernández (2017) aportan que este tipo de método nació en la enseñanza del derecho, pero ha tenido gran auge en la medicina y en otras áreas de la ingeniería.

Es importante señalar que también existe la tendencia del aprendizaje basado en proyectos, el cual tiene una perspectiva próxima al aprendizaje basado en problemas, en cuanto al papel activo y colaborativo que deberá asumir el estudiantado ante los proyectos.

\section{Pedagogías emergentes}

Para Gros (2015), algunas de las características de las pedagogías emergentes son el resultado de la sociedad del conocimiento e implican:

Repensar el significado de la educación y, muy especialmente, hay que tener presente que para que realmente la persona pueda dirigir sus procesos formativos requiere desarrollar importantes capacidades que no se generan de forma espontánea. La formación sigue siendo necesaria pero no solo orientada a lo disciplinar sino, especialmente, a capacitar a las personas para pasar por los distintos espacios sin tener una experiencia fragmentada, dispersa y un exceso de carga emocional y cognitiva. (p. 62)

En el contexto de las pedagogías emergentes, como una forma de integrar y relacionar áreas disciplinares, Chinyowa, Mwakapenda y Mokuku (2017) brindan un referente integrador entre contenidos matemáticos y artes, por medio del estudio de un caso cotidiano, el referente es importante, ya que las pedagogías emergentes promueven la relación de diferentes elementos y prácticas participativas para la resolución de casos.

Sobre la contribución de las pedagogías emergentes a la educación, Adell y Castañeda (2015) plantean: "Las pedagogías emergentes son como organismos en evolución, en estado de 'llegar a ser', sujetas a influencias de su contexto y a adaptaciones y cambios no predecibles de antemano" (p. 2). Para Gros (2015), cuatro aspectos tendientes a una pedagogía emergente deben ser considerados en las estrategias de formación: "la personalización, el aprendizaje activo, el aprendizaje colaborativo y el aprendizaje autónomo o autodirigido" (p. 63). Como se puede notar, las pedagogías emergentes resultan en una tendencia que 
promueve principios conocidos en cuanto al aprendizaje activo y participativo; todo esto relacionado con el uso de las TIC y, particularmente, el e-learning resultan un contexto propicio para su aplicación.

Para Domingo (2013), el papel de la experiencia debe verse apoyada por el reconocimiento, en lo pedagógico, de una gama amplia de saberes y relaciones útiles para docentes. Lo apuntado por estos autores tiene injerencia en el proceso de enseñanza-aprendizaje. Resulta curioso que profesionales sin formación en lo educativo y que se desempeñan en este campo, logran aplicar principios pedagógicos, con base, únicamente, en la experiencia docente; estos van surgiendo de manera natural en el acto educativo, lo mismo ocurre en la aplicación de las TIC en los procesos de enseñanza, pues algunas veces el perfil tecnológico profesional propicia la innovación y la implementación de dinámicas que promueven el trabajo colaborativo.

Una de las premisas de las pedagogías emergentes es que el rol estudiantil varía, las actividades propuestas son tendientes a respetar sus inclinaciones, contexto y experiencias previas para que pueda construir sus aprendizajes y experiencias con la guía docente. A partir de esta premisa, López, Calvo y García (2016) apuntan que "ya que no es suficiente con presentar una información para que ésta sea aprendida, sino que el alumno o alumna debe reconstruirla a lo largo de su propia experiencia interna" (p. 6).

Por otra parte, el rol docente en el escenario del e-learning implica, desde el enfoque de las pedagogías emergentes, actividades de aprendizaje situadas en el contexto del estudiantado, donde lo cotidiano sea relacionado con los contenidos. También las actividades colaborativas serán de gran valor, pues requieren coordinación grupal, asumir roles, negociación, cooperación, ejes importantes que brindarán competencias valiosas para el mundo laboral. El estudiantado deberá asumir un rol participativo y valioso en cuanto a valorar el papel propio y el de otras personas. Con esto, ¿cómo actúa el personal docente?, la perspectiva de facilitador es el mejor sinónimo para ubicar el papel que asumirá, con roles de guía, orientador, motivador, necesarios para que el estudiantado sea activo y no un ente pasivo en los procesos académicos.

Una orientación particular es brindar referentes explicativos para la elaboración de las actividades planteadas (mapas conceptuales, cuadros comparativos, esquemas) para lo cual se pueden proponer videos o tutoriales que expliquen cómo elaborarlos, así también, lejos 
de ser actividades individuales, puede convertirse en actividades colaborativas, donde cada estudiante pueda aportar su conocimiento y experiencias previas, en este punto el personal docente facultado como facilitador, hará un monitoreo de la labor de los grupos colaborativos: envío de mensajes, seguimiento, motivación y corrección en caso de divergencia con respecto al objetivo de cada actividad.

La idea de esta transformación en los procesos académicos es reforzada por Onye y Yunfei (2016): "La tecnología está poniendo a prueba diferentes filosofías pedagógicas, y se ha convertido en un crisol pulir tanto el contenido como el contexto de los canales educativos de entrega, independientemente de las diferencias demográficas" (p. 327). Esto refuerza el papel democratizador de la educación que pueden favorecer las TIC; sin embargo, el uso debe ser planificado en términos del público meta, necesidades y el tipo de mediación pedagógica que se utilizará.

Con respecto a lo anterior y a la utilización cautelosa de las TIC, rescatamos una investigación realizada por Geer, White, Zeegers, Wing y Barnes (2017), en relación con el uso de dispositivos móviles. Advierten que la introducción de nuevas tecnologías debe estar acompañada por una nueva pedagogía. Como resultado de la investigación, al realizar un cambio pedagógico, se obtuvo una mayor colaboración, comunicación, autosuficiencia del alumnado y el desarrollo de experiencias auténticas. Su perspectiva no coloca las tecnologías de la información y comunicación (TIC) sobre lo pedagógico, sino tiende a modificar prácticas pedagógicas por medio de las posibilidades de la tecnología; esto es parte de los principios de las pedagogías emergentes.

Desde la perspectiva de Ricaurte (2016), las nuevas pedagogías deben considerar el aprendizaje en red con una componente de cooperación, apertura y una sociedad del conocimiento. Un referente pedagógico de interés es el aportado por Silva (2017), quien presenta un modelo centrado en las e-actividades con la siguiente premisa:

El modelo se basa en una propuesta metodológica de aprendizaje centrada en el estudiante, que se establece en la convicción de que los sujetos aprenden "haciendo" e "interactuando". Desde este punto de vista, se fomenta el desarrollo de "actividades" que privilegien el trabajo práctico, en equipo y relacionado con situaciones concretas orientadas a la resolución de problemas o el desarrollo de habilidades de orden superior. (p. 8) 
Por medio de un modelo centrado en las e-actividades, el rol docente es facilitar, moderar y orientar; debe plantear actividades que permitan la reflexión, así también debe estar muy pendiente de consultas y del avance en el caso de trabajos colaborativos. Esta visión sobre la importancia de las e-actividades es reafirmada por Mora y Madriz (2017), como parte de una experiencia en un grupo de cursos virtuales, donde identificaron la importancia de este elemento para el éxito académico del estudiantado y, cómo, para el correcto diseño de las e-actividades, el profesorado requiere de acompañamiento técnico pedagógico. Realmente dentro de las pedagogías emergentes las e-actividades deben, además de propiciar la colaboración, poseer los principios del aprendizaje basado en proyectos y problemas, además de una evaluación participativa que evalúe para aprender.

Las e-actividades son mucho más provechosas cuando se fomenta el aprendizaje situado o contextual, tal como lo señalan Castillo y Cerón (2015), quienes orientan sobre el propósito de este tipo de actividades al "reactivar la memoria, recordar el proceso de aprendizaje y la experiencia real vivida; lo que contribuye significativamente a reforzar nuevos conocimientos" (p. 91).

Dentro de la línea de personalismo educativo - tema que desarrollaremos en el siguiente apartado-que enuncian las pedagogías emergentes, el personal tutor virtual debe considerar el perfil tecnológico de ingreso del estudiantado para valorar posibles adaptaciones y guías con el fin de facilitar la participación en diferentes actividades propuestas en el e-learning.

\section{Personalismo educativo}

El personalismo educativo resulta una tendencia tratada por Víctor García Hoz (1985), en su obra Educación personalizada. Este autor es considerado su principal exponente; más de tres décadas después, algunos principios que enuncia siguen vigentes, más aún, con el avance de las tecnologías y el uso de la virtualidad por medio del e-learning y espacios de interacción, pueden ser potenciados desde una perspectiva flexible y consecuente con los nuevos modelos de enseñanza y, en particular, por medio de las pedagogías emergentes. 
El aprendizaje personalizado o personalismo educativo, consiste en estrategias de aprendizaje, soluciones e intervenciones que se ajustan a los objetivos individuales del estudiante y tienen en cuenta las diferencias de origen del conocimiento, la pasión o interés en los temas, y el dominio de la materia. El propósito del aprendizaje personalizado es capacitar a los estudiantes para tomar posesión de la experiencia de aprendizaje y prepararse a sí mismos para el aprendizaje permanente. (Johnson, Adams, Cummins, Estrada, Freeman y Hall, 2016, p. 28)

Para Balduzzi (2015), el personalismo educativo implica un rol de liderazgo por parte de los sujetos actores del proceso educativo, a partir de lo cual el profesorado debe participar activamente en este y sus metas educativas.

Para Feu (2017), este tipo de aprendizaje se justifica porque "hace falta que el alumno que crece se confronte con los problemas, se cuestione, se contraste con la vida de los demás para poder medir la realidad de su propia vida" (p. 231), esta orientación es tendiente al uso del aprendizaje basado en problemas (ABP).

Para comprender mejor este concepto, es deseable delimitar a la persona como un ser social y en el contexto, esto permite tomar en cuenta elementos en los procesos educativos:

Desde el punto de vista social la persona se integra en una comunidad, en la que cada uno disfruta de un nombre y en la cual se le reconoce su lugar, de manera que se convierte en única e insustituible. La comunidad es construida por las relaciones recíprocas de los unos con los otros. La finalidad de la comunidad se encuentra en ella misma y no puede ser conseguida sin que las personas la consigan también. (Feu, 2017, p. 213-214)

El aprendizaje personalizado ya ha sido motivo de estudio en el ámbito internacional para las políticas educativas.

La UNESCO está estableciendo precedentes importantes para dirigir las discusiones internacionales y sesiones de formulación de políticas. Con anterioridad al Foro de Política Mundial de alto nivel, de la UNESCO 2015, los líderes de la educación de 53 
países fueron encuestados sobre cómo lograr un mejor aprendizaje abierto, en línea, y flexible. Más del 50\% identificó la importancia de la función docente en el apoyo a los estudiantes a través de aprendizaje personalizado. (p. 28)

Como vemos, el aprendizaje personalizado fomenta un rol estudiantil activo, empoderado y, a su vez, ubica al personal docente como facilitador.

Actualmente existen algunos otros estudios que plantean su uso. Lara (2015) trata la temática en la legislación educativa de Ecuador desde esta perspectiva; Fernández (2011), con una reflexión sobre cómo el personalismo propicia la formación del ser humano y la relación con la comunidad; Choin (2015), Herrera, Tapia y Sánchez (2015) dejan entrever cómo resulta una temática importante a nivel internacional y que por medio del uso de las TIC y el e-learning se ve potenciado.

Aunado a lo anterior, una orientación significativa sobre la importancia del personalismo educativo resulta del prestigioso informe Horizon del 2016, publicado por The New Media Consortium, el cual retoma el principio de la personalización educativa, con el fin de contextualizar sus potencialidades en el modelo del e-learning.

Tal como lo aporta Leadbetter (2008, citado por Wright, 2015, p. 3 ), es "poner al alumno en el centro del sistema educativo". Complementando el principio anterior, el aprendizaje personalizado implica:

Ampliar los conceptos educativos de la diferenciación (enseñanza adaptado a las preferencias de aprendizaje de los diferentes alumnos) y la individualización (la enseñanza las necesidades de aprendizaje de los diferentes alumnos) para conectar con el interés y las experiencias del satisfacer las necesidades, capacidades e intereses de cada estudiante.

Tomando algunos principios aportados por Williams (2013, citado por Wright, 2015), desde la personalización del aprendizaje podemos promover ambientes de aprendizaje efectivo por medio de las siguientes estrategias: "conocer a los estudiantes como aprendices; colaboración y el uso efectivo de las TIC" (p. 4). Esto último requiere una infraestructura adecuada y plataformas que permitan las actividades y recursos. Se debe tener presente que el personalismo educativo 
beneficia al estudiantado en cuanto toma en consideración los estilos de aprendizaje. Estos principios son actualmente tratados sobre las bases del diseño universal para el aprendizaje (DUA), que toma en cuenta, en el diseño de contenido, que la accesibilidad debe considerar todo tipo de población y evitar dificultades para ciertas poblaciones (Alhawiti y Abdelhamid, 2017).

Como vemos, existe un importante fundamento para poder trasladar los principios de la personalización educativa en el e-learning, lo cual no es más que una expresión de la educación a distancia basado en el uso intensivo de las tecnologías de la información y comunicación (TIC). Vera (2016), refiriéndose a la corriente del personalismo, plantea que esta "concentra sus esfuerzos en torno a la comprensión profunda del ser humano, expresada a través de comportamientos concretos en relación con el medio, la sociedad, la vida vivida de los hombres, en su condición humana". (p. 10). En una investigación realizada por esta misma autora en el contexto de educación superior de Paraguay y como parte de una problemática en la educación superior de ese país, señala:

La mirada desde la óptica del Personalismo es fundamental si se quiere construir una sociedad auténticamente humana, productiva, transformadora, emancipada. La demanda de la sociedad en esta era digital es la creatividad, la autonomía, la autogestión, la cogestión, el autoconocimiento, la innovación, entre otros, y la Pedagogía Personalista es esencial para que el egresado pueda insertarse de forma exitosa en esta cambiante sociedad. (pp. 5-6)

Entre las últimas tendencias que apoyan el personalismo educativo están las analíticas de aprendizaje (Zapata, 2013), herramientas que permiten la toma de decisiones en cuanto a recursos y actividades planteadas en el e-learning. Las analíticas de aprendizaje resultan una tendencia que puede posibilitar identificar perfiles en actividades del estudiantado, formato de presentación de la información, son flexibles para el uso de diferentes entornos de aprendizaje (Coll, Engel y Niño, 2017).

Finalmente, en cuanto al e-learning y sus potencialidades para el personalismo educativo, Sfenrianto y Hasibuan (2017) reiteran estas posibilidades, pero advierten cómo este proceso no se viene dando en la virtualidad o bien no es una práctica común, pues aún requiere la mejora de la mediación pedagógica en este tipo de espacios. Es 
precisamente la posibilidad de ajustar actividades a las preferencias y necesidades del estudiantado, las que hacen que el e-learning sea un terreno fértil para tal fin.

\section{Ecologías del aprendizaje}

Con el advenimiento de las pedagogías emergentes, el personalismo educativo y otras tendencias en el ámbito educativo, surge el término de las ecologías del aprendizaje, concepto enmarcado dentro de una metáfora denominada ecosistemas digitales, el cual brinda algunas pautas sobre cómo el aprendizaje se ve modificado por la interacción con las TIC.

La ecología del aprendizaje deja entrever los retos que se plantean a la educación escolar y formal y las transformaciones que es necesario atender, dando paso a la personalización del aprendizaje entendida como la diversificación de las oportunidades, experiencias y recursos en función de las necesidades e intereses de los aprendices. (Alcántar, 2017, p. 6)

En relación con el concepto de ecosistemas digitales, Islas y Carranza (2017) plantean que recibe una caracterización relativa con sus diferentes elementos:

En los últimos años los dispositivos tecnológicos e internet con sus aplicaciones y herramientas han tenido alto impacto en distintos ámbitos entre ellos el educativo, por lo que surge el interés de presentar este acercamiento al estado del conocimiento respecto a los ecosistemas digitales y su manifestación en el aprendizaje. (p. 2)

Indican que "los ecosistemas digitales de aprendizaje representan un paradigma de los futuros sistemas de educación, soportados en las tecnologías de la información y las comunicaciones" (p. 5).

Los fragmentos anteriores dan cuenta de cómo la disponibilidad de las tecnologías actuales va modificando las prácticas educativas. 


\section{Evaluar para aprender}

La evaluación de los aprendizajes resulta un elemento clave en los procesos educativos, particularmente dentro de las pedagogías emergentes. Con un cambio de paradigma en el rol docente y actividades de aprendizaje, sufre algunas transformaciones hacia una modalidad evaluativa que implica participación y donde el estudiantado se involucra durante todo el proceso. Estas son algunas de las premisas de la tendencia de evaluar para aprender, algunas veces conocida como evaluación formativa, como lo señala Sonsoles (2017), la cual aporta también otras denominaciones como evaluación formadora, auténtica, dinámica o continua. Una forma sencilla de entender la tendencia de evaluar para aprender (Dorrego, 2016) tiene que ver con que el profesorado confiera el rol evaluativo al estudiantado.

Como lo aporta Vidal (2017), un proceso final que debe asumir la evaluación es su propia realimentación para efectos de las mejoras en la enseñanza aprendizaje. Por otra parte, para Salas y Salas (2017), al referirse a algunos principios de la evaluación para el aprendizaje, señalan que tienen que ver con reconocer el propósito evaluativo en estudiantes y docentes tanto como componente integral, claridad, criterios y estándares, efecto motivador, y aportar realimentación.

\section{Metodología}

Para esta investigación se utilizó el método hermenéutico dialéctico, el cual consiste en una metodología cualitativa basada en la interpretación de información. La hermenéutica requiere un esfuerzo interpretativo con el fin de lograr profundidad en el análisis de la temática deseada (Ortiz, (2015).

Como forma de abordaje y con el fin de mejorar la aproximación conceptual definida en esta investigación, se recurrió a más de 60 fuentes bibliográficas para apoyar las temáticas incluidas en la investigación, las cuales fueron recuperadas de sitios académicos (Google académico), revistas científicas y bases de datos en particular de EBSCO, en su mayoría artículos, tanto en idioma inglés como en español, la búsqueda se limitó a un plazo máximo de antigüedad de cinco años, con el fin de contar con referentes actualizados, aunque en algunos casos, por la pertinencia de los aportes, se incluyeron fuentes aún anteriores al periodo señalado. 
Para el método hermenéutico dialéctico, se recomienda "usar la frase o la oración como unidad de análisis, iniciar el proceso con la lectura cuidadosa de los textos, dividiéndolos en unidades temáticas y aplicando la respectiva codificación" (Cárcamo 2005, citado por Villalobos 2017, p. 365). Con esta premisa metodológica, se identificaron categorías bien definidas y con representantes comunes durante el periodo bibliográfico definido para el análisis. Además, la metodología utilizada se orientó por la línea de la triangulación teórica aportada por Atencio (2015), con el fin de verificar los resultados obtenidos por medio de diferentes fuentes documentales y, así, validar la información y aportes localizados.

La evidencia de la relación entre las pedagogías emergentes se encontraron en el personalismo educativo, aprendizaje colaborativo, diseño centrado en el estudiantado, aprendizaje ubicuo, aprendizaje basado en proyectos, aprendizaje basado en problemas, ecologías del aprendizaje y evaluar para aprender; algunas de estas categorías son presentadas en la tabla 1.

\section{Tabla 1}

Presencia de categorías de pedagogías emergentes según los estudios analizados

\begin{tabular}{|c|c|c|c|c|}
\hline Autorías & $\begin{array}{l}\text { Personalismo } \\
\text { educativo }\end{array}$ & $\begin{array}{l}\text { Aprendizaje } \\
\text { colaborativo }\end{array}$ & $\begin{array}{c}\text { Aprendizaje } \\
\text { ubicuo }\end{array}$ & $\begin{array}{c}\text { Aprendizaje } \\
\text { contextual o situado }\end{array}$ \\
\hline 1. De Corte (2015) & $\cdot$ & $\cdot$ & & $\cdot$ \\
\hline $\begin{array}{l}\text { 2. Alhawiti y } \\
\text { Abdelhamid (2017) }\end{array}$ & - & & & - \\
\hline $\begin{array}{l}\text { 3. Shah, Attid y ur } \\
\text { Rheman (2017) }\end{array}$ & - & & - & \\
\hline $\begin{array}{l}\text { 4. Velazco, Abuchar, } \\
\text { Castilla y Rivera } \\
\text { (2017) }\end{array}$ & & - & - & \\
\hline $\begin{array}{l}\text { 5. Sfenrianto y } \\
\text { Hasibuan (2017) }\end{array}$ & - & & & \\
\hline $\begin{array}{l}\text { 6. IIgaz y Gulbahar } \\
\text { (2017) }\end{array}$ & - & & & \\
\hline 7. Díez y Díaz (2018) & & • & • & - \\
\hline $\begin{array}{l}\text { 8. Quintero y Romero } \\
(2018)\end{array}$ & & & - & - \\
\hline 9. Lara (2015) & - & & & - \\
\hline
\end{tabular}




\begin{tabular}{|c|c|c|c|c|}
\hline Autorías & \begin{tabular}{|c|} 
Personalismo \\
educativo
\end{tabular} & $\begin{array}{l}\text { Aprendizaje } \\
\text { colaborativo }\end{array}$ & $\begin{array}{l}\text { Aprendizaje } \\
\text { ubicuo }\end{array}$ & $\begin{array}{c}\text { Aprendizaje } \\
\text { contextual o situado }\end{array}$ \\
\hline 10. Choin (2015) & $\cdot$ & & & • \\
\hline $\begin{array}{l}\text { 11. Herrera, Tapia y } \\
\text { Sánchez (2015) }\end{array}$ & $\cdot$ & & & $\cdot$ \\
\hline $\begin{array}{l}\text { 12. Johnson, Adams, } \\
\text { Cummins, Estrada, } \\
\text { Freeman y Hall (2016) }\end{array}$ & $\bullet$ & & & • \\
\hline 13. Wright (2015) & - & & & $\cdot$ \\
\hline 14. Balduzzi (2015) & $\cdot$ & & & \\
\hline 15. Vera (2016) & $\bullet$ & & & $\cdot$ \\
\hline 16. Zapata (2013) & • & & • & \\
\hline $\begin{array}{l}\text { 17. Geer, White, } \\
\text { Zeegers, Wing y } \\
\text { Barnes (2017) }\end{array}$ & & $\cdot$ & $\cdot$ & $\cdot$ \\
\hline 18. Ricaurte (2016) & & • & & \\
\hline 19. Silva (2017) & $\cdot$ & $\cdot$ & & • \\
\hline $\begin{array}{l}\text { 20. Roca, Reguant y } \\
\text { Canet (2015) }\end{array}$ & & • & & $\cdot$ \\
\hline 21. Gros (2015) & $\cdot$ & $\cdot$ & $\bullet$ & $\cdot$ \\
\hline $\begin{array}{l}\text { 22. Hernández, } \\
\text { González y Muñoz } \\
(2015)\end{array}$ & & $\cdot$ & & $\cdot$ \\
\hline $\begin{array}{l}\text { 23. Castillo y Cerón } \\
(2015)\end{array}$ & & $\cdot$ & $\cdot$ & $\cdot$ \\
\hline $\begin{array}{l}\text { 24. Velandia, Serrano } \\
\text { y Martínez (2017) }\end{array}$ & & & $\bullet$ & $\bullet$ \\
\hline 25. Dorrego (2016) & & - & & • \\
\hline
\end{tabular}

Nota: Elaboración propia. El orden no alfabético de las fuentes en la tabla corresponde al momento de análisis durante la investigación hermenéutica realizada.

De la tabla anterior es posible notar la coincidencia entre autores y autoras en relación con los componentes que permiten ubicar las pedagogías emergentes, como aquellas que promueven el uso de las TIC hacia una personalización o personalismo educativo y el rol más activo para el estudiantado, donde el aprendizaje colaborativo cobrará un papel relevante. Se puede apreciar, además, la coincidencia, donde 
Gros (2015), de Corte (2015), Díez y Díaz (2018), Geer, White, Zeegers, Wing y Barnes (2017), Castillo y Cerón (2015) son los principales representantes de esta tendencia, pues cumplen con la presencia de tres o más categorías analizadas, lo cual les convierte en referentes muy precisos para el estudio realizado.

\section{Conclusiones y recomendaciones}

A partir de esta investigación se puede concluir que el e-learning, por su naturaleza y potencialidades, resulta el campo justo para el desarrollo de pedagogías emergentes, por cuanto se tiene una convergencia en recursos de las TIC, aunado a las posibilidades de interacción y colaboración.

El e-learning permite la posibilidad de contar con una pluralidad en cuanto al perfil del personal tutor, que en su práctica pedagógica debe poseer competencias en el uso de las tecnologías para favorecer el aprendizaje ubicuo, la aplicación del aprendizaje colaborativo y actividades situadas (contextuales); todas estas categorías presentes en las pedagogías emergentes para que pueda responder a las necesidades y características de la población meta en un contexto favorable para su aplicación, de manera tal que promueven un aprendizaje activo y participativo de sus estudiantes con la utilización de recursos como las TIC.

También se debe considerar que para la mediación es necesario contemplar el perfil de ingreso del estudiantado, y todas aquellas ayudas que permitan el desarrollo eficaz de los procesos académicos, por cuanto se basa en una propuesta centrada en el alumnado. Diagnósticos previos pueden brindar información importante al personal docente para la toma de decisiones en cuanto al tipo de atención, actividades y formatos de contenidos más propicios.

Las pedagogías emergentes tienden al desarrollo de competencias por parte del individuo; por medio de actividades significativas, situadas y colaborativas, se podrán mejorar los procesos de formación, para contar con un individuo capaz de insertarse y enfrentar los retos del mundo laboral. Al respecto, las TIC son un campo donde ocurren con mayor facilidad, por las posibilidades comunicativas y de interacción, tanto de manera sincrónica como asincrónica.

En este contexto, las actividades propuestas deben respetar intereses, necesidades, contextos y experiencias previas para que el 
estudiantado pueda construir sus aprendizajes y experiencias con la guía docente a partir de sus logros a través de su propia experiencia; lo cotidiano debe ser relacionado con los contenidos, sin dejar de lado que las actividades colaborativas requieren de coordinación grupal, asignación de roles, técnicas de negociación que le proporcionan al estudiantado competencias valiosas para el mundo laboral.

El e-learning facilita la personalización del aprendizaje, por las posibilidades tecnológicas, aunado a un rol más activo por parte del estudiantado. Por este medio surgen tendencias de aprendizaje colaborativo y aprendizaje basado en problemas (ABP), dado que se trata de metodologías más activas y donde el estudiantado puede inclinarse por orientaciones disciplinares de su interés particular o de su contexto.

La tendencia de evaluar para aprender cobra gran importancia dentro de las pedagogías emergentes, ya que en sus premisas es posible encontrar el rol participativo estudiantil, en cuanto al involucramiento en la evaluación; esto es vital para el desarrollo de trabajos colaborativos y actividades contextuales.

El rol docente dentro de las pedagogías emergentes resultan de gran importancia y es más bien el de facilitador, pues es quien planea el proceso académico y la mediación de los contenidos, pudiendo propiciar que el estudiantado desarrolle actividades significativas, contextuales (basadas en problemas cotidianos o ABP) y compartiendo con sus pares, por medio del aprendizaje colaborativo.

Para futuros estudios es importante la profundización en el aporte de las analíticas de aprendizaje y algunas otras tecnologías emergentes, como lo son las tecnologías del aprendizaje adaptativo, ligadas con los principios del personalismo educativo y por su contribución para la mediación en las pedagogías emergentes; estas resultaron dos categorías que surgen en algunas fuentes bibliográficas analizadas en la investigación realizada.

\section{Referencias}

Adell, J., y Castañeda, L. (2015). Las pedagogías escolares emergentes. Cuadernos de pedagogía, 462, 21-25. Recuperado de https://digitum.um.es/xmlui/bitstream/10201/49329/1/2015cuader.a.pdf

Alhawiti, M., y Abdelhamid, Y. (2017). A Personalized e-Learning Framework. Journal of Education And E-Learning Research, 4(1), 
15-21. Recuperado de http://search.ebscohost.com/login.aspx?direct $=$ true $\& \mathrm{db}=$ eric \& AN=EJ1148435\&lang $=$ es\&site $=$ ehost-live

Area, M., y Guarro, A. (2012). La alfabetización informacional y digital: Fundamentos pedagógicos para la enseñanza y el aprendizaje competente. Revista española de documentación científica, 35(Monográfico), 46-74. doi: 0.3989/redc.2012.mono.977. Recuperado de http://redc.revistas.csic.es/index.php/redc/article/ view/744/825

Atencio, E. (2015). Gestión del conocimiento y medición del capital intelectual como recurso intangible en las organizaciones que aprenden. Análisis comparativo desde sus modelos originarios. CICAG, 13(1), 223-234. Recuperado de http://publicaciones. urbe.edu/index.php/cicag/article/view/3926/5120

Avello, R., y Duart, J. (2016). Nuevas tendencias de aprendizaje colaborativo en e-learning: Claves para su implementación efectiva. Estudios pedagógicos (Valdivia), 42(1), 271-282. Recuperado de https://scielo.conicyt.cl/scielo. php?pid=S0718-07052016000100017\&script $=$ sci_arttex

Badia, A., Meneses, J., y García, C. (2018). La identidad del profesor como docente virtual: Roles, enfoques y sentimientos. El caso de la Universitat Oberta de Catalunya (UOC). Informe de investigación. Recuperado de http://femrecerca.cat/meneses/files/badia_meneses_garcia_es_2017.pdf

Balduzzi, E. (2015). Liderazgo educativo del profesor en el aula y la personalización educativa. (Spanish). Revista Española de Pedagogía, 73(260), 141-155. Recuperado de http://search.ebscohost. com/login .aspx?direct=true $\& \mathrm{db}=$ eue $\& A N=101375792 \&$ lan$\mathrm{g}=$ es\&site $=$ ehost-live

Camacho, I., Gómez, M., y Pintor, M. (2015). Competencias digitales en el estudiante adulto trabajador. Revista Interamericana de Educación de Adultos, 37(2). Recuperado de http://www.redalyc. org/pdf/4575/457544924002.pdf

Castillo, S. y Cerón, A. (2015). Aprendizaje situado para la formación de competencias investigativas en el estudiante de psicología clínica: Análisis desde el pensamiento complejo. Revista Internacional de Evaluación y Medición de la Calidad Educativa, 2(1). Recuperado de http://journals.epistemopolis.org/index.php/ calidadeducativa/article/view/989/553 
Cervantes, M., y Saker, A. (2016). Importancia del uso de las plataformas virtuales en la formación superior para favorecer el cambio de actitud hacia las TIC. Estudio de caso: Universidad del Magdalena, Colombia. Revista Iberoamericana de Evaluación Educativa, 6(1). Recuperado de https://revistas.uam.es/index.php/ riee/article/view/3847

Chinyowa, K., Mwakapenda, W., y Mokuku, S. (2017). Crossing the River: Towards an emerging pedagogy for integrating arts and mathematics education. Mosenodi, 20(1), 48-56. Recuperado de http://journals.ub.bw/index.php/mosenodi/article/view/996/607

Choin, D. (2015). El personalismo: Una propuesta de acción para la generación del pensamiento libre y la construcción del saber de los estudiantes de lengua y literatura. Academia, 14, 327-336. Recuperado de https://www.saber.ula.ve/handle/123456789/41293

Coll, C., Engel, A., y Niño, S. (2017). La actividad de los participantes como fuente de información para promover la colaboración. Una analítica del aprendizaje basada en el modelo de Influencia educativa distribuida. Revista de Educación a Distancia, 53. Recuperado de http://revistas.um.es/red/article/view/289501/210431

De Corte, E. (2015). Aprendizaje constructivo, autorregulado, situado y colaborativo: Un acercamiento a la adquisición de la competencia adaptativa (matemática). Páginas de Educación, 8(2), 1-35. Recuperado de http://www.scielo.edu.uy/pdf/pe/v8n2/v8n2a01.pdf

De Miguel, M. (2015). Ideología y pedagogía empírica: Cuestiones para un debate. RIE: Revista De Investigación Educativa, 33(2), 269-287. doi:10.6018/rie.33.2.226611. Recuperado de http://search.ebscohost.com/login.aspx?direct=true $\& \mathrm{db}=$ fua\& $\mathrm{AN}=103596371 \&$ lang $=$ es \& site $=$ ehost-live

Díez, E. y Díaz, J. (2018). Ubiquitous learning ecologies for a critical cyber-citizenship. Comunicar, 26(54), 49-58. doi:10.3916/ C54-2018-05. Recuperado de http://search.ebscohost.com/login. aspx ?direct $=$ true $\& \mathrm{db}=$ eue $\& \mathrm{AN}=127255242 \&$ lang $=$ es \& site $=\mathrm{e}-$ host-live

Domingo, J. (2013). El saber de la experiencia en la formación inicial del profesorado. Revista interuniversitaria de formación del profesorado, 78, 125-136. Recuperado de https://dialnet.unirioja.es/ servlet/articulo? codigo $=4688508$ 
Dorrego, E. (2016). Educación a distancia y evaluación del aprendizaje. Revista de Educación a Distancia, 50 (12), 1-20. Recuperado de $\mathrm{http} / / /$ revistas.um.es/red/article/view/271241

Fernández, M., y Valverde, J. (2013). Comunidades de práctica: Un modelo de intervención desde el aprendizaje colaborativo en entornos virtuales. Comunicar, 21 (42), 97-105. Recuperado de http:// www.redalyc.org/html/158/15830197011/

Fernández, M. (2016). Modelo educativo emergente en las buenas prácticas TIC. Revista Fuentes, 18(1), 33-47. Recuperado de https:// idus.us.es/xmlui/handle/11441/53840

Fernández, M. (2011). Actualidad educativa del personalismo de Xavier Zubiri. Estudios sobre educación, 21, 83-100. Recuperado de https://www.unav.edu/publicaciones/revistas/index.php/ estudios-sobre-educacion/article/view/4423

Feu, T. (2017). Fundamentos filosóficos de una pedagogía personalista. Revista de fomento social, 286, 203-253. Recuperado de https:// dialnet.unirioja.es/servlet/articulo?codigo $=6075995$

Flores, Á., y García, A. (2017). Sistema de aprendizaje ubicuo en ambientes virtuales. Revista Cubana de Educación Superior, 36(2), 27-37. Recuperado de http://search.ebscohost.com/login.aspx?direct $=$ true $\& \mathrm{db}=$ fua $\& A N=126117211 \&$ lang $=$ es \&site=ehost-live García, V. (1985). Educación personalizada. Madrid: Editorial Rialp.

Geer, R., White, B., Zeegers, Y., Wing, A., y Barnes, A. (2017). Emerging pedagogies for the use of iPads in schools. British Journal of Educational Technology, 48(2), 490-498. doi:10.1111/ bjet.12381. Recuperado de http://search.ebscohost.com/login. aspx?direct=true $\& \mathrm{db}=\mathrm{ehh} \& \mathrm{AN}=121348510 \&$ lang $=\mathrm{es} \&$ site $=\mathrm{e}-$ host-live

Giroux, H. (2003). Pedagogía crítica. Recuperado de http://online.aliat. edu.mx/adistancia/filosofia/U7/Lecturas/Pedagogia critica.pdf

Gros, B. (2015). La caída de los muros del conocimiento en la sociedad digital y las pedagogías emergentes. Teoría de la educación. Educación y Cultura en la Sociedad de la Información, 16(1), 58-68. doi:10.14201/eks20151615868. Recuperado de http://search.ebscohost.com/login.aspx?direct=true\&$\mathrm{db}=$ fua\&AN $=102126854$ \&lang $=$ es\&site $=$ ehost-live

Herrera, X., Tapia, M., y Sánchez, X. (2015). El personalismo en las aulas: El papel de los docentes en la transmisión de valores. 
Academia, 14, 278-285. Recuperado de erevistas.saber.ula.ve/ index.php/academia/article/view/7599

Hernández, N., González, M., y Muñoz, P. (2015). El rol docente en las ecologías de aprendizaje: Análisis de una experiencia de aprendizaje colaborativo en entornos virtuales. Revista de currículum y formación del profesorado, 19(2). Recuperado de http://digibug. ugr.es/bitstream/10481/37369/1/rev192ART9.pdf

Ilgaz, H., y Gulbahar, Y. (2017). Why do learners choose online learning: the learners' voices. International Conference On E-Learning, 130-136. Recuperado de http://search.ebscohost.com/ login.aspx?direct $=$ true $\& \mathrm{db}=$ asn $\& A N=127022859 \&$ lang=es\&site $=$ ehost-live

Islas, C., y Carranza, M. (2017). Ecosistemas digitales y su manifestación en el aprendizaje: Análisis de la literatura. RED - Revista de Educación a Distancia, 55, 1-13. doi:10.6018/red/55/9. Recuperado de http://search.ebscohost.com/login.aspx?direct=true\&$\mathrm{db}=$ fua\&AN $=127079586 \&$ lang $=$ es\& site $=$ ehost-live

Jenaro, C., Flores, N., Poy, R., González Gil, F., y Martínez, E. (2013). Metodologías docentes en la educación superior: Percepciones del profesorado sobre su importancia y uso. Recuperado de $\mathrm{http}: / /$ gredos.usal.es/jspui/bitstream/10366/123297/1/art_2.pdf

Jiménez, K. (2010). Educación, pedagogía, didáctica y currículo. Universidad de Costa Rica: Departamento de Docencia Universitaria.

Johnson, L., Adams, S., Cummins, M., Estrada, V., Freeman, A., y Hall, C. (2016). NMC Informe Horizon 2016 Edición Superior de Educación. Austin, Texas: The New Media Consortium. Recuperado de http://cdn.nmc.org/media/2016-nmc-horizon-report-HE-ES.pdf

Lara, F. (2015). Personalismo en la legislación educativa ecuatoriana: Una aproximación desde el pensamiento de Gabriel Marcel. Academia, 14, 230-238. Recuperado de https://www.saber.ula.ve/ bitstream/123456789/41273/3/articulo24.pdf

López, S., Calvo, J., y García, C. (2016). Evaluación de una experiencia de innovación docente para el aprendizaje significativo en pedagogía: Un marco para la reflexión a partir de la percepción de los estudiantes. Estudios sobre educación, 18, 229-254. Recuperado de https://www.unav.edu/publicaciones/revistas/index.php/ estudios-sobre-educacion/article/view/4673/4030 
Martín, M., Díaz, E., Sánchez, J. (2015). Coordinación interdisciplinar mediante aprendizaje basado en problemas. Una aplicación en las asignaturas dirección de producción y estadística empresarial. Revista de Investigación Educativa, 33(1), 163-178. Recuperado de http://revistas.um.es/rie/article/view/179741/170851

Mora, F., y Madriz, S. (2017). E-Actividades: Una experiencia práctica en las asignaturas virtuales de la Cátedra de Ciencias Penales. XVII Congreso Internacional Innovación y Tecnología en Educación a Distancia. Recuperado de http://repositorio.uned.ac.cr/ reuned/bitstream/120809/1694/1/texto4.pdf

Mora, F., y Hooper, C. (2016). Trabajo colaborativo en ambientes virtuales de aprendizaje: Algunas reflexiones y perspectivas estudiantiles. Revista Electrónica Educare, 20(2). Recuperado de http://www.redalyc.org/pdf/1941/194144435020.pdf

Morales, S. y Munévar, P. (2014). Hacia una convergencia entre las tecnologías emergentes y las pedagogías emergentes. Revista de investigaciones UNAD, 13(2). Recuperado de http://repository. unad.edu.co/bitstream/10596/6857/1/005vol13num2.pdf

Moreira, J., Reis, A., y Machado, A. (2017). La educación superior a distancia y el e-Learning en las prisiones en Portugal. Сотиnicar, 25(51), 39-49. Recuperado de http://www.redalyc.org/ pdf/158/15850360004.pdf

Onye, U., y Yunfei, D. (2016). Digital natives and digital divide: analysing perspective for emerging pedagogy. Proceedings of The IADIS International Conference on Cognition \& Exploratory Learning in Digital Age, 324-328. Recuperado de http://search.ebscohost.com/login.aspx?direct=true $\& \mathrm{db}=\mathrm{eu}-$ e\&AN=120267024\&lang $=$ es\&site $=$ ehost-live

Ortiz, G. (2015). Hermenéutica analógica, verdad y método. Diánoia, 60(74), 155-163. Recuperado de http://www.scielo.org.mx/pdf/ dianoia/v60n74/v60n74a8.pdf

Parra, J., Amariles, M., y Castro, C. (2016). Aprendizaje basado en problemas en el camino a la innovación en ingeniería. Ingenierías USBMed, 7(2), 96-103. Recuperado de http://revistas.usb.edu. co/index.php/IngUSBmed/article/view/2486

Quintero, J., y Romero, S. (2018). Aprendizaje ubicuo: M-learning como una fortaleza en el uso de las TIC. Revista Internacional de Tecnología, Conocimiento y Sociedad, 6(1), 13-19. Recuperado 
de http://search.ebscohost.com/login.aspx?direct=true $\& \mathrm{db}=$ as$\mathrm{n} \& \mathrm{AN}=127007550 \&$ lang $=$ es\&site $=$ ehost-live

Roblizo, M., y Cózar, R. (2015). Usos y competencias en TIC en los futuros maestros de educación infantil y primaria: Hacia una alfabetización tecnológica real para docentes. Pixel-Bit. Revista de Medios y Educación, 47, 23-39. Recuperado de http://www.redalyc.org/html/368/36841180002/

Roca, J., Reguant, M., y Canet, O. (2015). Aprendizaje basado en problemas, estudio de casos y metodología tradicional: Una experiencia concreta en el grado en enfermería. Procedia-Social and Behavioral Sciences, 196, 163-170. Recuperado de https://www. sciencedirect.com/science/article/pii/S187704281504015X

Rodríguez, C., y Fernández, J. (2017). Evaluación del aprendizaje basado en problemas en estudiantes universitarios de construcciones agrarias. Formación universitaria, 10(1), 61-70. Recuperado de https://scielo.conicyt.cl/pdf/formuniv/v10n1/art07.pdf

Ricaurte, P. (2016). Pedagogies for the open knowledge society. International Journal of Educational Technology in Higher Education, 13(1), 1-10. doi:10.1186/s41239-016-0033-y. Recuperado de http://search.ebscohost.com/login.aspx?direct=true \&db=ehh\&AN=117761472\&lang $=$ es\&site $=$ ehost-live

Salas, R. y Salas, A. (2017). Evaluación para el aprendizaje en ciencias de la salud. Edumecentro, 9(1), 208-227. Recuperado de http:// scielo.sld.cu/pdf/edu/v9n1/edu13117.pdf

San Martín, Á. (2009). Incertidumbre ante las pedagogías emergentes. Teoría de la Educación. Educación y Cultura en la Sociedad de la Información, 10(1). Recuperado de http://www.redalyc.org/ pdf/2010/201018023001.pdf

Sfenrianto, y Hasibuan, Z. (2017). Step-Function Approach for E-Learning Personalization. Telkomnika, 15(3), 1362-1367. doi:10.12928/TELKOMNIKA.v15i3.4457. Recuperado de $\quad \mathrm{http} / / /$ search.ebscohost.com/login.aspx?direct=true\&d$\mathrm{b}=\mathrm{a} 9 \mathrm{~h} \& \mathrm{AN}=125915486 \&$ lang $=$ es\& $\&$ ite $=$ ehost-live

Shah, H., Attiq, S., y ur Rehman, S. (2017). Role of E-Learning in National Development. Journal of Managerial Sciences, 11(1), 145156. Recuperado de http://search.ebscohost.com/login.aspx?direct $=$ true $\& \mathrm{db}=\mathrm{bth} \& \mathrm{AN}=125229288 \&$ lang $=$ es\&site=ehost-live 
Silva, J. (2017). Un modelo pedagógico virtual centrado en las E-actividades. Revista de Educación a Distancia, 53, 1-20. Recuperado de http://revistas.um.es/red/article/view/290021

Sonsoles, M. (2017). Evaluación y aprendizaje. MarcoELE: Revista de Didáctica Español. Lengua Extranjera, 24, 1-43. Recuperado de http://marcoele.com/descargas/24/fernandez-evaluacion_aprendizaje.pdf

Suárez, J. M., Almerich, G., Díaz, I., \& Fernández, R. (2012). Competencias del profesorado en las TIC: Influencia de factores personales y contextuales. Universitas Psychologica, 11(1), 293-309. Recuperado de http://www.scielo.org.co/scielo. php?script $=$ sci_abstract\&pid $=$ S1657-92672012000100024

Torres, C., y Alcántar, M. (2017). Ecosistemas digitales y su manifestación en el aprendizaje: Análisis de la literatura. Revista de Educación a Distancia, 55, 1-13. Recuperado de http://www.um.es/ ead/red/55/islas_carranza.pdf

Velandia, C., Serrano, F., y Martínez, M. (2017). La investigación formativa en ambientes ubicuos y virtuales en educación superior. Comunicar: Revista Científica de Comunicación y Educación, 25(51), 09-18. Recuperado de https://www.revistacomunicar. $\mathrm{com} /$ verpdf.php?numero $=51 \&$ articulo $=51-2017-01$

Velazco, S., Abuchar, A., Castilla, I., y Rivera, K. (2017). E-learning: Rompiendo fronteras. Revista Electrónica Redes de Ingeniería, 91-100. Recuperado de http://search.ebscohost.com/login.aspx?direct $=$ true $\& \mathrm{db}=$ fua $\& A N=125534778 \&$ lang $=$ es\&site $=$ ehost-live

Vera, O. (2016). Pedagogía personalista para la transformación de la educación superior. ACADEMO Revista de Investigación en Ciencias Sociales y Humanidades, 3(2). Recuperado de http:// revistacientifica.uamericana.edu.py/index.php/academo/article/ view/90

Vidal, J. (2017). Evaluar para aprender. Padres y Maestros/Journal of Parents and Teachers, 370, 12-17. Recuperado de https:// revistas.upcomillas.es/index.php/padresymaestros/article/ view/7855/7645

Villalobos, L. (2017). Enfoques y diseños de investigación social: Cuantitativos, cualitativos y mixtos. San José de Costa Rica: EUNED. 
Wright, J. (2015). Personalised learning: An overview. Recuperado de http://itali.uq.edu.au/filething/get/1865/Personalised_learning overview_Final_16_Mar_15.pdf

Zapata, M. (2013). Analítica de aprendizaje y personalización. Campus virtuales, 2(2), 88-118. Recuperado de http://uajournals.com/ojs/ index.php/campusvirtuales/article/view/41/40 
